\title{
Physical consequences of mass singularities
}

\author{
H.F. Contopanagos \\ Institute for Theoretical Physics, State University of New York, Stony Brook, NY 11794-3840, USA
}

and

\section{M.B. Einhorn}

Institute for Theoretical Physics, University of California, Santa Barbara, CA 93106-4030, USA ${ }^{1}$ and Randall Laboratory of Physics, University of Michigan, Ann Arbor, MI 48109-1120, USA

Received 12 November 1991; revised manuscript received 22 December 1991

\begin{abstract}
We argue that the perturbation expansion of massless field theories are uniquely and unambiguously defined for properly formed observables. In so doing, we generalize the Kinoshita-Lee-Nauenberg theorem to show that there are no observable "mass-shell anomalies". Paradoxically, in realistic circumstances, massive electrons give rise to non-intuitive, enhanced effects, for example, an unexpected background to the search for right-handed charged currents at HERA.
\end{abstract}

Singularities associated with massless particles have found an uneasy coexistence with perturbation theory. These singularities are of two varieties: (1) infrared singularities associated with massless particles of zero energy, or (2) collinear singularities associated with the degeneracy between massless, on-shell particles travelling in identical directions. The "infrared catastrophe" associated with the masslessness of the photon is believed not to be a problem, owing to the Bloch-Nordsieck theorem [1,2] that the sum over experimentally degenerate final states is free of singularities associated with the masslessness of the photon. On the other hand, the failure of this theorem in non-abelian gauge theories $[3,4]$ and its implications have been much debated. While it was shown that the inclusion of initial-state degeneracy restorted the cancellation of divergences [5], this does not establish whether physical observables always must involve such initial-state degeneracy, and there seems to be a divergence of opinion on this score.

The Kinoshita-Lee-Nauenberg (KLN) theorem $[6,7]$ is a generalization of the Bloch-Nordsieck result proving, modulo certain subtleties having to do with renormalization, the non-occurrence of mass singularities in certain averaged or inclusive quantities involving degenerate states. However, although universally accepted since its elaboration over 25 years ago, this theorem leaves certain questions unresolved. For example, Lee and Nauenberg [7] pointed out a paradox in QED associated with the fact that, owing to a collinear singularity, the total probability for an electron to emit forward bremsstrahlung while undergoing helicity flip remains finite in the limit that the electron mass tends to zero. If correct, this would have profound implications, for it would mean that chiral symmetry was not restored in the massless limit. Another illustration of this phenomenon is that if, instead of the electron, one gives the photon a mass so that chirality is preserved, one finds that the forward bremsstrahlung of a longitudinal photon does not vanish in the limit that the photon mass tends to zero [8]. Thus, it would seem that massless QED is inherently ambiguous, its predictions appearing to depend on the method of regu-

\footnotetext{
1 Address until August 1992.
} 
larization and the symmetries enforced, a kind of infrared anomaly $\# 1$. [9].

The situation is even more confused for nonabelian theories, especially in applications to quantum chromodynamics (QCD). Indeed, people performing radiative corrections frequently cite the inapplicability of the KLN theorems to non-abelian gauge theories $[4,10]$, and there remain uncompensated logarithmic dependences on light quark masses in these radiative corrections as traditionally calculated. It has been argued that, since the initial-state cancellation required by the KLN theorem would require a very special relationship between the quark structure function and the two-particle, quark-gluon structure function, the cancellation should not be expected to occur [4]. This confusion is of some practical import, not only because of how radiative corrections are to be calculated, but also because it renders suspect the factorization of hard hadronic processes into a non-perturbative, long-distance contribution and a perturbative, short-distance contribution. This factorization is undermined by the appearance of mass singularities, a long-distance phenomenon, in the perturbative evaluation of quark-gluon $S$-matrix elements.

These issues provide ample motivation to take up this old subject once more and to attempt to resolve these matters. In a series of papers [11-14] we have examined these issues and attempted to resolve the conceptual puzzles. In this letter, we shall summarize our conclusions; for mathematical details, we refer the reader to our longer papers.

By way of application, we shall point out that these matters are relevant to the search for right-handed currents in deeply inelastic electron scattering, especially at HERA [11]. As a result, there is a previously unnoticed electromagnetic background of order $\alpha$. When polarized electron beams become available at HERA, it is intended to search for interactions of right-helicity electrons of the form $e_{R}^{-} p \rightarrow v_{R}+X$, where, experimentally, $v_{R}$ simply represents no electron in the detector, i.e., a veto on neutral currents. If there were a right-handed charged current, $v_{R}$ would correspond to a right-handed neutrino. In the SM, there are no right-handed charged currents, so the rate

\#1 We shall refer to these effects as "mass-shell anomalies," inasmuch as they do not occur in off-shell Green's functions. for this is expected to be extremely small. Since the electron is not massless, helicity and chiral eigenstates differ slightly, and the rate for the SM charged current interaction is not precisely zero, but, compared to the rate observed for a left-helicity electron $\mathrm{e}_{\mathrm{L}}^{-}$, it should be down by a factor of order $\left(m_{\mathrm{e}} / E\right)^{2}$, where $m_{\mathrm{e}}$ and $E$ are the mass and energy of the electron. For HERA, this ratio is about $4 \times 10^{-10}$ and presents a negligible rate. So observation of any charged current interaction of a right-handed electron would be of great significance ${ }^{\# 2}$. However, consider the reaction $e_{R}^{-} p \rightarrow v_{e L} \gamma+X$. The right-handed electron may emit a photon, flip its helicity, and undergo a normal weak interaction. If the photon proceeds down the beam pipe, it will go undetected, so this reaction presents a background for the nonradiative process. The amplitude for bremsstrahlung of a photon accompanied by helicity flip is proportional to $m_{\mathrm{e}}$, so one would naively expect this rate to be quite negligible, of order $\alpha\left(m_{\mathrm{e}} / E\right)^{2}$, times the usual rate for a charged-current weak interaction. However, because of a collinear singularity associated with forward emission of the photon, the calculated rate is only suppressed by a factor of $\alpha$, a dramatic consequence of the original observation of Lee and Nauenberg [7]. One of the consequences of our work is to resolve this paradox and to show that, strictly speaking, in the limit $m_{\mathrm{e}} \rightarrow 0$, the observable rate would in fact vanish. Remarkably, it turns out that, for the physically relevant parameters, the naive calculation is very nearly correct, and the rate is only suppressed by a factor of $\alpha$ !

Returning to the general theory, the presence of mass singularities is evidence that the usual Feynman-Dyson $S$-matrix $S_{\mathrm{FD}}$ does not exist. This is because the free hamiltonian $H_{0}$ does not describe the asymptotic states in theories with massless particles ${ }^{\# 3}$. However, rather than modify the hamiltonian, the traditional approach has been either to sum incoherent cross sections [1] which we shall refer to as the "cross-section method", or to seek "coherent" states [17] between which $S_{\mathrm{FD}}$ will be free of mass singularities. The latter view was justified and exten-

\#2 All the more so since the CDF collaboration reports [15] a lower limit on a new charged vector boson of $520 \mathrm{GeV}$, beyond the range of sensitivity of the HERA experiment.

\#3 Apparently, the first person to identify this as the origin of the infrared catastrophe was Friedrichs [16]. 
sively developed by Kibble [18] and, later, by Zwanziger [19], who developed an LSZ-type reduction formalism. The close connection between coherent states and the observation of Friedrichs [16] was made more transparent by development of an alternate $S$-matrix in a number of special cases [20-23]. This stems from the supposition that, starting with a hamiltonian $H$ describing the interaction of one or more massless particles, one can find asymptotic hamiltonians $H_{\mathrm{A}}$ describing asymptotic states for which the corresponding $S$-matrix $S_{\mathrm{A}}$ does exist, i.e., is free of mass singularities $\# 4$. A common characteristic of such hamiltonians $H_{\mathrm{A}} \equiv H_{\mathrm{A}}(\Delta)$ is that they depend on parameters $\Delta$ not present in the original theory $H$, so that the interpretation of $S_{\mathrm{A}} \equiv S_{\mathrm{A}}[\Delta]$ requires further discussion. While it is not necessary in principle, for any given observable, it is extremely convenient to choose such parameters to coincide with the experimental resolutions characteristic of the particular measurement being considered.

As an illustration, consider the interaction in QED,

$$
V_{\mathrm{I}}^{(\mathrm{QED})}(t)=e \int \mathrm{d}^{3} x: \bar{\Psi}^{(\mathrm{e})}(x) \gamma^{\mu} \Psi^{\mathrm{e}}(x): A_{\mu}(x),
$$

where the operators are in the interaction picture, i.e., they have free field time dependence. After Fourier transforming and expressing the fields in terms of creation and annihilation operators, we obtain eight terms involving absorption or emission of photons, electrons, or positrons which can be abbreviated as

$$
\begin{aligned}
& V^{(\mathrm{QED})}(t)=e \int \frac{\mathrm{d}^{3} k_{1}}{\sqrt{(2 \pi)^{3} 2 \omega\left(\boldsymbol{k}_{1}\right)}} \frac{\mathrm{d}^{3} k_{2}}{\sqrt{(2 \pi)^{3} 2 \omega\left(\boldsymbol{k}_{2}\right)}} \\
& \quad \times \sum_{l=1}^{8} h_{l}\left(\boldsymbol{k}_{1}, \boldsymbol{k}_{2}, \boldsymbol{k}_{\gamma}\right) \exp \left[-\mathrm{i}(\mathscr{S} \omega)^{l} t\right]
\end{aligned}
$$

where the exponent involves sums or differences of the energies associated with the quanta involved in the vertex $h_{l}$. For example, a typical vertex is

\footnotetext{
\#4 This was proved for potential theory in ref. [21] and for the Pauli-Fierz model in ref. [22], but has only been demonstrated for certain processes and cases in QED in ref. [23].
}

$$
\begin{aligned}
h_{1} & =\sum_{\alpha_{1} \alpha_{2 \lambda} \lambda}\left(\frac{(2 \pi)^{3}}{2 \omega\left(\boldsymbol{k}_{\gamma}, m_{\gamma}\right)}\right)^{1 / 2} \\
& \times b_{\alpha_{1}}^{\dagger}\left(\boldsymbol{k}_{1}\right) b_{\alpha_{2}}\left(\boldsymbol{k}_{2}\right) a_{\lambda}\left(\boldsymbol{k}_{\gamma}\right) \bar{U}^{\alpha_{1}}\left(\boldsymbol{k}_{1}\right) \boldsymbol{\phi}_{\lambda}\left(\boldsymbol{k}_{\gamma}\right) U^{\alpha_{2}}\left(\boldsymbol{k}_{2}\right),
\end{aligned}
$$

where the $b_{\alpha}(k)\left(a_{\lambda}(k)\right)$ are the usual annihilation operators for electrons (photons) of definite helicity and momenta, $U^{\alpha}(k)$ are Dirac spinors, and $\epsilon_{\lambda}$ are the photon polarization vectors. (For the complete explicit expression, see ref. [13].) Mass singularities are either of infrared type, arising when the photon momentum $\boldsymbol{k}_{\boldsymbol{\gamma}}$ vanishes, or, for massless electrons, of collinear type, arising from vertices in which the threemomenta at the vertex are parallel, configurations for which the phase factor $(\mathscr{S} \omega)^{l}$ vanishes. To give an example of an acceptable asymptotic hamiltonian, one may define the modified interaction

$$
\begin{aligned}
& V_{\AA}^{(\mathrm{QED})}(\Delta ; t)=e \int \frac{\mathrm{d}^{3} k_{1}}{\sqrt{(2 \pi)^{3} 2 \omega\left(\boldsymbol{k}_{1}\right)}} \frac{\mathrm{d}^{3} k_{2}}{\sqrt{(2 \pi)^{3} 2 \omega\left(\boldsymbol{k}_{2}\right)}} \\
& \quad \times \sum_{l=1}^{8} \Theta_{\Delta}\left(\boldsymbol{k}_{i}\right) h_{l}\left(\boldsymbol{k}_{1}, \boldsymbol{k}_{2}, \boldsymbol{k}_{\gamma}\right) \exp \left[-\mathrm{i}(\mathscr{P} \omega)^{l} t\right] .
\end{aligned}
$$

Here we have simply inserted an unspecified function of the momenta $\boldsymbol{\Theta}_{\Delta}\left(\boldsymbol{k}_{i}\right)$. Its purpose is to include the infrared and collinear contributions. For these configurations, it must reproduce $V^{(\mathrm{QED})}$ exactly, so, in the massless limit, the $\Theta$ function must take the value one for the precisely collinear $\left(\boldsymbol{k}_{1} \propto \boldsymbol{k}_{2}\right)$ or infrared $\left(\omega_{3}=0\right)$ configurations. An example of the sort of function we have in mind is $\Theta\left(\Delta_{l}-\left|(\mathscr{S} \omega)^{l}\right|\right)$, where $\Theta(x)$ denotes the usual step-function, and $\Delta_{t}$ are in general a set of arbitrary parameters but most conveniently identified with the experimental resolutions. The full asymptotic hamiltonian is taken to be $H_{\mathrm{A}}(\Delta) \equiv H_{0}+V_{\mathrm{A}}$, where $H_{0}$ is the usual free hamiltonian.

More abstractly, the idea is to find a hamiltonian, $H_{\mathrm{A}}$, and associated states $\left|\psi_{\mathrm{A}}^{ \pm}\right\rangle$so that, for any (normalizable) state $|\psi\rangle$, its time evolution $\exp (-\mathrm{i} H t)|\psi\rangle$ approaches $\exp \left(-\mathrm{i} H_{\mathrm{A}} t\right)\left|\psi^{ \pm}\right\rangle^{\mathrm{A}}$ as $t \rightarrow \mp \infty$. To state the same thing more precisely, it is assumed that $H_{\mathrm{A}}$ can be found so that the Møller wave 
operators $\left.\Omega_{H, H_{A}}^{ \pm}\right)_{\text {exist as }}$ (proper) unitary operators in Fock space $\mathscr{H}_{\mathrm{F}}$, i.e. ${ }^{\$ 5}$,

$\Omega_{H, H_{\mathrm{A}}}=\lim _{t \rightarrow \mp \infty} \exp (\mathrm{i} H t) \exp \left(-\mathrm{i} H_{\mathrm{A}} t\right)$.

The utility of these Møller operators is that they provide the correspondence between an arbitrary state $|\psi\rangle$ and its associated in- and out-states $\# 6\left|\psi^{ \pm}\right\rangle^{\mathrm{A}}$, viz., $|\psi\rangle=\Omega_{H, H_{\mathrm{A}}}^{ \pm}\left|\psi^{ \pm}\right\rangle^{\mathrm{A}}$. If the theory described by $H$ only involves massive particles, then the free hamiltonian $H_{0}$ is one such admissible $H_{\mathrm{A}}$, but this is usually not the case when the theory contains one or more massless particles. The corresponding $S$-matrix $\# 7$

$S_{\mathrm{A}} \equiv \Omega\left(\hat{H}_{H, H_{\mathrm{A}}}^{\dagger} \Omega\left(\hat{H}_{H, H_{\mathrm{A}}}^{+}\right.\right.$,

is associated with transitions between an arbitrary in-state $\left|\psi^{+}\right\rangle^{\mathbf{A}}$ and out-state $\left|\phi^{-}\right\rangle^{\mathbf{A}}$, viz., ${ }^{\mathrm{A}}\left\langle\phi^{-} \mid \psi^{+}\right\rangle^{\mathrm{A}} \equiv^{\mathrm{A}}\left\langle\phi^{+}\left|S_{\mathrm{A}}\right| \psi^{+}\right\rangle^{\mathrm{A}}$. It is this $S$-matrix $S_{\mathrm{A}}$ that is expected to be a proper unitary operator, or, more physically stated, this $S$-matrix has matrix elements in Fock space $\mathscr{H}_{\mathrm{F}}$ that are free of any mass singularities. Although the existence of such singularityfree transition operators has not been proved in general, there is substantial evidence that such asymptotic hamiltonians do indeed exist. Since the perturbative Feynman-Dyson $S$-matrix $S_{\mathrm{FD}}$ in gauge theories has mass singularities, we know that the free hamiltonian $H_{0}$ is not in this class. However, if the massless theory is somehow regulated, such as by giving the vector boson a mass or dimensionally regulating by continuation to $4+\epsilon$ dimensions [ 25,26$]$, then, of course, in the regulated theory, the modified free hamiltonian $H_{\delta}^{(\epsilon)}$ becomes acceptable. But, while operators that are well-defined for the massless theory are approached smoothly as the regulator $\epsilon$ is re-

\#5 To be mathematically precise, we assume strong convergence to the limit. In general, the asymptotic hamiltonian $H_{\mathrm{A}}$ may depend explicitly on time, as was done in ref. [23], the formalism is a bit simpler if we restrict our attention to timeindependent $H_{\mathrm{A}}$.

*6 Lest there be any confusion with non-Fock asymptotic states employed in the literature $[18,23]$, we emphasize that our physical Hilbert space $\mathscr{H}_{\mathrm{F}}$ is a conventional Fock space, and these asymptotic states necessarily are in the same $\mathscr{H}_{\mathrm{F}}$ since $\Omega_{H} \pm H_{\mathrm{A}}$ are unitary operators within this space.

$\# 7$ A pedagogical review of scattering theory in this language may be found in chapters 6 and 7 of Newton [24]. While this reference does not treat the massless case, his formal discussion carries over with the replacement of $H_{0}$ by $H_{\mathrm{A}}$. moved, certain other quantities such as the usual Feynman-Dyson $S$-matrix $S_{\mathrm{FD}}^{(\epsilon)}$ diverge.

It is a simple exercise to relate $S$-matrices corresponding to different asymptotic hamiltonians, since the asymptotic states are simply related by a unitary change of basis. For the regulated theory, one may relate $S_{\mathrm{A}}^{(\epsilon)}$ to $S_{\mathrm{FD}}^{(\epsilon)}$ :

$S_{\mathrm{A}}^{(\epsilon)}=\Omega_{H_{\AA}^{(\epsilon)}, H_{\delta}^{(\epsilon)}}^{(-)} S_{\mathrm{FD}}[H]^{(\epsilon)} \Omega_{H^{(\epsilon)}, H_{\delta}^{(\epsilon)}}^{(+)}$,

where $S_{\mathrm{FD}}[H]^{(\epsilon)}$ is the usual Feynman-Dyson $S$ matrix

$S_{\mathrm{FD}}[H]^{(\epsilon)} \equiv \Omega_{H^{(\epsilon)}, H_{0}^{(\epsilon)}}^{(-)^{\dagger}} \Omega_{H^{(\epsilon)}, H_{0}^{(\epsilon)}}^{(+)}$.

The relation (7) is closely related to the definition of coherent states. If $|\psi\rangle$ is any Fock state, then

$|\psi ; \pm\rangle_{\mathrm{coh}}^{\mathrm{A},(\boldsymbol{\epsilon})} \equiv \Omega_{H_{\lambda}^{(\epsilon)}, H_{\delta}^{(\epsilon)}}^{( \pm \dagger}|\psi\rangle$

defines the associated coherent states $|\psi ; \pm\rangle_{\mathrm{coh}}^{\mathrm{A},(\epsilon)}$. By definition, the Fock space matrix elements of $S_{\AA}^{(\epsilon)}$ may be written as

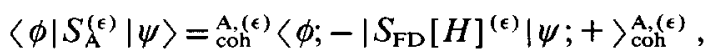

for any two Fock states $|\phi\rangle,|\psi\rangle$. In other words, the desired transition amplitudes, the Fock space matrix elements of $S_{A}^{(\epsilon)}$ that tend to finite limits as $\epsilon \rightarrow 0$, may be thought of as the matrix elements of the usual Feynman-Dyson $S$-matrix $S_{\mathrm{FD}}[H]^{(\epsilon)}$ between coherent states $[17,18]^{\# 8}$.

For the case of scattering of an electron in an external field, we have shown by explicit calculation [13] of the lowest non-trivial radiative corrections that the evaluation of the single-particle matrix element $\left\langle\mathrm{e}^{\prime}\left|S_{\mathrm{A}}[\Delta]\right| \mathrm{e}\right\rangle$ reproduces the classic results obtained by the cross-section method, for example, in ref. [7]. This correspondence results if (and only if) one identifies the parameters $\Delta$ in the asymptotic hamiltonian with the experimental energy and angular resolutions. It was established in ref. [12] that (a) the same result is obtained if one uses either mass regularizations for the electron and photon or if one uses dimensional regularization and that $(b)$ the re-

\#8 Although these coherent states are frequently described as the asymptotic states dressed by soft and collinear particles, in fact one can show [13] that, in the limit $\epsilon \rightarrow 0$, these coherent states are orthogonal to the true asymptotic scattering states, so such an interpretation is misleading. 
sult is non-singular in the limit that the regulators are removed. This gives us confidence in the conclusions for the novel applications below.

What if we had chosen $H_{\mathrm{A}}(\Delta)$ with parameters $\Delta$ not equal to the experimental resolution? The foregoing discussion applies to both massless quanta and to particles having a small but finite mass. (For massive particles, choosing $\Delta$ sufficiently small removes any possibility of degeneracy.) Thus, for sufficiently small $\Delta, S_{\mathrm{A}}[\Delta]$ reduces to $S_{\mathrm{FD}}$. If this choice of $\Delta$ is small compared to the experimental resolutions, then, clearly, the cross-section method must be used to construct the observable cross section. This observation generalizes as follows: If the choice of asymptotic hamiltonian $H_{\mathrm{A}}(\Delta)$ involves parameters $\Delta$ that are smaller than the observational degeneracy, one must add to the elastic cross section, the inelastic contributions of soft and collinear quanta that the measurement cannot resolve. More precisely stated, if $\Delta_{\exp }$ denotes the experimental resolution, and $\Delta<A_{\text {exp }}$, then

$$
\begin{aligned}
& \mathrm{d} \sigma_{\text {obs }}\left(\Delta_{\text {exp }}\right)=\left|\left\langle l^{\prime}\left|S_{\mathrm{A}}\left[\Delta_{\text {exp }}\right]\right| l\right\rangle\right|^{2} \\
& \quad=\left|\left\langle l^{\prime}\left|S_{\mathrm{A}}[\Delta]\right| l\right\rangle\right|^{2}+\sum_{\mathrm{i}, \mathrm{f}}\left|\left\langle\mathrm{f}\left|S_{\mathrm{A}}[\Delta]\right| \mathrm{i}\right\rangle\right|^{2},
\end{aligned}
$$

where the second term represents a sum over those inelastic initial and final states that cannot be experimentally distinguished from the elastic contribution. This formula has been explicitly verified in the case of scattering of an electron in an external field. \#9 The necessity to include initial-state degeneracy differs from the Bloch-Nordsieck [1] result for abelian theories. However, it is well known to be required generally for collinear singularities $[7,25]$ and for infrared singularities in non-abelian theories in those amplitudes involving non-singlet initial states $[3,4]$.

Another way to understand the significance of the parameters $\Delta$ in the asymptotic hamiltonian is as follows: The parameters $\Delta$ may be thought of a specifying a degeneracy domain within which particles are either sufficiently soft or sufficiently collinear. Then one can show that all matrix elements of $S_{\mathrm{A}}[\Delta]$ between states having quanta lying within the degenerate phase space vanish [13]! This makes it clear why, when one chooses $\Delta$ equal to the experimental reso-

\footnotetext{
\#9 See appendix B of ref. [13].
}

lution, the $S_{\mathrm{A}}$-matrix is completely characterized by those matrix elements having only non-degenerate particles in the initial and final states.

In most applications, the initial-state resolutions and final-state resolutions differ, and it is not necessary to choose the same asymptotic hamiltonian to describe the initial and final states. Indeed, it is simpler to define a modified scattering matrix

$S_{A^{\mathrm{f}} A^{\mathrm{i}}}\left[\Delta^{\mathrm{f}}, \Delta^{\mathrm{i}}\right] \equiv \Omega_{H, H_{\mathrm{Af}}}^{\left(-H_{\mathrm{f}}^{+}\right.} \Omega_{H, H_{A^{\mathrm{i}}}}^{+}$,

where $H_{\mathrm{A}^{\mathrm{f}}}$ is associated with out-states and $H_{\mathrm{A}^{\mathrm{i}}}$ with in-states. For abelian theories, so long as the initialstate energy resolution $\Delta E_{\mathrm{i}}$ is less than the detector resolution, as is normally the case, then the matrix element will in fact be independent of $\Delta E_{\mathrm{i}}$. However, this is not true for collinear singularities, and the matrix element is not independent of the initial-state angular resolution $\delta \theta_{\mathrm{i}}$ or, alternatively, the initial-state transverse momentum resolution. (We shall defer for the moment a discussion of how that resolution is to be experimentally determined.) Generally, for massive particles, the cutoff on the collinear singularity is governed not simply by the angular resolution $\delta_{\theta}^{\text {in }}$ or the corresponding transverse momentum resolution $\delta p_{\perp}$, but rather by the so-called transverse mass $m_{\perp}=\sqrt{p_{\perp}^{2}+m^{2}}$ or corresponding angle $m_{\perp} / E=$ $\sqrt{\delta_{\theta}^{\text {in } 2}+\chi_{\mathrm{e}}^{2}}$, where $\chi_{\mathrm{e}} \equiv m / E$. This is the quantity that sets the scale of the cutoff on the collinear singularity. Thus, if $\delta_{\theta}^{\text {in }} \gg \chi_{\mathrm{e}}$, then the mass may be neglected, whereas, if $\delta_{\theta}^{\text {in }} \ll \chi_{\mathrm{e}}$, it is the mass rather than the resolution that is the important parameter. In this latter case, treating the incoming state as a single particle state and neglecting the experimental resolutions is a valid approximation.

Now let us return to the paradox posed earlier concerning helicity-flip bremsstrahlung in the forward direction. A straightforward application of the preceding formalism $[11,14]$ leads to the result that the cross section for $e_{R}^{-} p \rightarrow v_{e} \gamma+X$ is proportional to the corresponding cross section for $e_{L}^{-} p \rightarrow v_{e L}+X$ times the factor

$\alpha \chi_{\mathrm{e}}^{2} \int_{\delta_{\theta}^{\text {in }}}^{\Delta_{\theta}^{\mathrm{f}}} \frac{\mathrm{d} \theta^{2}}{\left(\chi_{\mathrm{e}}^{2}+\theta^{2}\right)^{2}}$,

where $\Delta_{\theta}^{\mathrm{f}}$ is the smallest angle outside of which we can be certain that no photon was emitted and $\delta_{\theta}^{\text {in }}$ is 
the initial-state angular resolution, presumed to be smaller than $\Delta_{\theta}^{\mathrm{f}}$. This has the properties advertised: In the limit that $\chi_{\mathrm{e}} \rightarrow 0$, this vanishes so that helicity conservation is restored for the massless theory. However, for usual experimental situations in which $\delta_{\theta}^{\text {in }} \lesssim \chi_{e} \ll \Delta_{\theta}^{\mathrm{f}}$, this is a term of $\mathrm{O}(\alpha)$ and not of order $\alpha \chi_{\mathrm{e}}^{2}$. Given the anticipated luminosity and degree of polarization expected for HERA, and given the validity of the CDF lower bound [15], any events observed with a right-handed $e_{\bar{R}}^{-}$would presumably be due to this background ${ }^{\# 10}$.

This helicity-flip bremsstrahlung is an example of what we have called an "evanescent" process, in that it involves a transition that naively appears to exist for the massless limit of the massive theory, but in fact does not exist in the massless theory. Despite their common origins, this is a different type of mass singularity than the more familiar infrared or collinear ones that manifestly diverge in the limit of zero mass. Evanescent processes remain perfectly finite in the massless limit of a massive theory, but in fact vanish when experimental resolutions are taken into account. What we have illustrated is a general result concerning such processes: the massless limit is smooth but subtle. Another application concerns the decoupling of longitudinal photons from massless electrons, or longitudinal gluons from massless quarks, a subject on which there has been some confusion [9]. Using this formalism, it is straightforward to show that these longitudinal modes decouple from observables [14].

Until now, although we asserted some knowledge of the magnitude of the experimental initial-state angular resolution, we have not explained how it is to be determined in a given situation. This is an unfamiliar concept and requires considerable discussion to do it justice $[13,14]$. One would think that a classical source could have arbitrarily precise resolution, given that there is an infinite amount of time to prepare the beam. In such a case, the initial-state resolution could be inferred from the geometry of the experiment and the quantum limits on the precision of a measurement resulting from the uncertainty principle. For example, an electron travels a finite distance in a finite time $T$ from where it emerges from

${ }^{\sharp 10}$ Complete derivations and numerical results have been given in ref. [ 111$]$. the accelerator to the intersection point and this, in turn, implies a minimum energy uncertainty $\Delta E \equiv$ $\hbar / T$. (For a hard photon and a sufficiently energetic electron, this can be shown to yield a non-zero $\delta_{\theta}^{\text {in }}$.) Similarly, the knowledge that the particle lies within a certain region in the transverse plane sets a lower limit on the transverse momentum resolution which may in turn be translated into an initial-state angular resolution. However, in practice, we believe the initial-state angular resolution is set by limiting factors in the accelerating structures themselves, associated with betatron oscillations that are excited by the quantum fluctuations in the emission of bremsstrahlung photons by a relativistic electron [27]. Such bremsstrahlung form a "searchlight" peaking at an angle $\chi_{\mathrm{e}}$ with respect to the initial direction of the electron. The electron's recoil, this inevitable jitter in the electron's direction due to random emission of photons, is the mechanism that drives the betatron oscillations and creates uncertainty in the electron's direction. Thus, we anticipate that, in an optimally designed accelerator, the initial-state angular resolution $\delta_{\theta}^{\text {in }}$ will be not so much smaller than $\chi_{e}$, although, since the probability of bremsstrahlung is rather small, the resolution may be smaller than this on the average. As we have seen, the relative size of $\delta_{\theta}^{\text {in }}$ and $\chi_{\mathrm{e}}$ is all-important in determining whether initial-state degeneracy or coherence is important in practice, so one must be more precise than this orderof-magnitude estimate. In refs. [13,14] we have evaluated this angle directly from the betatron amplitude and transverse emittance of the beam and have found that $\delta_{\theta}^{\text {in }} / \chi_{\mathrm{e}}$ is about 0.1 for SLC, $0.3-0.4$ for LEP, and about 0.16 for HERA. Since this ratio is squared in calculating corrections to initial-state collinear singularities, this has a small effect. Thus, it is the electron mass and not the accelerator parameters that serves as the cutoff on the physical singularity. In these cases, naive calculations with $S_{\mathrm{FD}} S$ matrix elements ought to be accurate to $10 \%$ or better when compared with the correct answer based on $S_{\mathrm{A}}[\Delta]$. In proposed future $\mathrm{e}^{-} \mathrm{e}^{+}$colliders, the demands for luminosity lead to even smaller estimates of $\delta_{\theta}^{\text {in }} / \chi_{\mathrm{e}}$. In this sense, higher energies do not approach the massless limit.

As a mathematical aside, the coherent state formalism $[18,23]$ has been underutilized, at least in elementary particle physics, because it seems to re- 
quire the introduction of unfamiliar concepts, such as a generalization of the Hilbert space of states to von Neumann space, to take into account unitarily non-equivalent representations of the canonical commutation relations. In this work, we have revived an observation originally due to Blanchard [22] that one option for treating massless particles is to continue to associate the physical space with Fock space and identify the more familiar states associated with non-interacting particles described by $H_{0}$ with a non-equivalent representation of the canonical commutation relations $\# 1$.

In summary, we have developed the interpretation of the $S$-matrix approach to mass singularities. This demonstrates that the summation over degenerate initial states involves neither an ensemble average nor a modification of quantum mechanics but is intrinsically necessary to form meaningful observables or a sensible $S$-matrix. One consequence of this work is that, for properly formed transition amplitudes and observable cross sections, perturbation theory can be used for massless QED. Although electric charge is screened in the limit that the electron mass is zero, in fact, the usual Feynman rules may be used for massless electrons to evaluate meaningful transitions, provided initial-state and final-state resolutions are taken into account. We have ignored questions of gauge invariance here but wish to note that, despite $H_{\mathrm{A}}$ not being gauge invariant, results obtained thus far are gauge invariant, suggesting that, generally, gauge invariance will be maintained up to terms that vanish with the resolutions.

There are many other potential applications of this formalism. As a practical matter, initial-state radiative corrections, crucial for interpreting experiments at accelerators such as LEP, can be performed more precisely and efficiently because one does not require a cancellation between divergent cross sections but can display transition amplitudes with integrands that are free of mass singularities. On the theoretical side, non-abelian theories are more complicated, espe-

$\# 11$ This is very much in keeping with the philosophy elaborated by Wightman [28] and the conventional view of observables. While we will not elaborate on this here, we wish to emphasize that the use of this formalism requires the introduction of no new principles into quantum field theory and that calculations may be performed in Fock space. cially because of the difficulties presented by confinement as in $\mathrm{QCD} \# 12$. Matrix elements must be factored into hadronic, long-distance pieces and shortdistance pieces, involving quarks and gluons, to which perturbative QCD may be applied. The existence of mass singularities in the Feynman-Dyson perturbation expansion of quark-gluon amplitudes undermines factorization and requires a certain amount of fancy footwork to keep things straight. One would expect that using the $S_{\mathrm{A}}$ matrix which is free of mass singularities would be simpler, but what parameters are to be associated with the resolution $\Delta$ ? This is a subject for further research, but we would hazard the guess that the energy resolution is to be associated with the characteristic scale $A_{\mathrm{QCD}}$ involved in the factorization into parton amplitudes and structure functions. The initial-state transverse momentum resolution for partons is presumably of order $\Lambda_{\mathrm{QCD}}$, from which can be deduced an initial-state angular resolution for "beams" of quarks or gluons and the finalstate angular resolution is presumably whatever convention is used to define a quark or gluon jet. This formalism might also illuminate the proper role of higher-twist or quark mass effects that are important for determining the approach to the asymptotic limit. It appears that this approach also resolves some of the technical complications associated with the interpretation of anomalies in the parton model $[29,30]$.

We are grateful to D.N. Williams for numerous discussions and for pointing out that one could choose to regard the physical subspace as Fock space rather than a von Neumann space. We wish to thank J.D. Bjorken for discussions and correspondence. We wish to thank D.R.T. Jones, A. Mueller, G. Sterman, and A.S. Wightman for helpful discussions as well. Portions of this work were supported by the Department of Energy and by the National Science Foundation (Grants No. PHY89-04035 and No. PHY91-08054.)

$\# 12$ QCD with more than 16 flavors is thought to have a perturbative phase resembling QED, and this formalism should go through essentially unchanged for the evaluation of perturbative transition amplitudes in this weak coupling phase.

\section{References}

[1] F. Bloch and A. Nordsieck, Phys. Rev. 52 (1937) 54; 
D.R. Yennie, S.C. Frautschi and H. Suura, Ann. Phys. (NY) 13 (1961) 379.

[2] J. Schwinger, Phys. Rev. 76 (1949) 290.

[3] R. Doria, J. Frenkel and J.C. Taylor, Nucl. Phys. B 168 (1980) 93.

[4] C. Di'Lieto et al., Nucl Phys. B 183 (1981) 223.

[5] A. Axelrod and C.A. Nelson, Phys. Rev. D 31 (1985) 2385; I. Ito, Prog. Theor. Phys. 65 (1981) 1466;

A. Andrasi et al., Nucl. Phys. B 182 (1981) 104;

C.A. Nelson, Nucl. Phys. B 186 (1981) 187

[6] T. Kinoshita, J. Math. Phys. 3 (1962) 650.

[7] T.D. Lee and M. Nauenberg, Phys. Rev. 133 (1964) B1549.

[8] M.B. Einhorn and D.R.T. Jones, report (July 1987), unpublished.

[9] A.S. Gorsky, B.L. Ioffe and A. Yu. Khodjamirian, Phys. Lett. B 227 (1989) 474;

A.S. Gorsky and B.L. Ioffe, Part. World 1 (1990) 144; A.S. Gorsky, preprint ITEP-89-155.

[10] M. Böhm and H. Spiesberger, Nucl. Phys. B 294 (1987) 1081.

[11] H.F. Contopanagos and M.B. Einhorn, preprint UM-TH89-09 (July 1989); revised version UM-TH-91-19 (September 1991).

[12] H.F. Contopanagos, Nucl. Phys. B 343 (1990) 571.

[13] H.F. Contopanagos and M.B. Einhorn, preprint UM-TH90-11, NSF-ITP-90-165i (October 1990), Phys. Rev. D, to be published.

[14] H.F. Contopanagos and M.B. Einhorn, preprint UM-TH90-12, NSF-ITP-90-166i, Phys. Rev. D, to be published.

[15] J. Huth, preprint FERMILAB-CONF-91-223-E (August 1991).
[16] K.O. Friedrichs, Commun. Pure Appl. Math. 5 (1952) 349.

[17] V. Chung, Phys. Rev. B 140 (1965) 1110.

[18] T.W. Kibble, J. Math. Phys. 9 (1968) 315; Phys. Rev. 173 (1968) 1527; 174 (1968) 1882; 175 (1968) 1624.

[19] D. Zwanziger, Phys. Rev. D 11 (1975) 3481, 3504.

[20] T. Murota, Prog. Theor. Phys. 24 (1960) 1109.

[21 ] J. Dollard, J. Math. Phys. 5 (1964) 729.

[22] Ph. Blanchard, Commun. Math. Phys. 15 (1969) 156.

[23] P.P. Kulish and L.D. Faddeev, Teor. Mat. Fiz. 4 (1970) 153 [Theor. Math. Phys. 4 (1971) 745].

[24] R.G. Newton, Scattering theory of waves and particles, 2nd Ed. (Springer, Berlin, 1982).

[25] R. Gastmans and R. Meuldermans, Nucl. Phys. B 63 (1973) 277;

T. Muta and C.A. Nelson, Phys. Rev. D 25 (1982) 2222.

[26] A. Andrasi and J.C. Taylor, Nucl. Phys. B 63 (1973) 277; W.J. Marciano, Phys. Rev. D 12 (1975) 3861.

[27] M. Sands, The physics of electron storage rings: an introduction, preprint SLAC-121, UC-28 (ACC.)

[28] A.S. Wightman, in: Proc. 1967 Intern. Conf. on Particles and fields, eds. C.R. Hagen et .l., (Interscience, New York, 1967).

[29] R.D. Carlitz, J.C. Collins and A.H. Mueller, Phys. Lett. B 214 (1988) 229;

R.L. Jaffe and A.V. Manohar, Nucl. Phys. B 337 (1990) 509 ;

A.V. Manohar, Phys. Rev. Lett. 66 (1991) 2684.

[30] H.F. Contopanagos, in preparation. 